\title{
The effects of Electroporation on Skin Impedance
}

\author{
Luke Davies $^{1,2}$ and Paul Chappell ${ }^{2}$ \\ ${ }^{1}$ Institute of Complex Systems and Simulation, University of Southampton, Southampton, \\ SO17 1BJ, UK \\ ${ }^{2}$ Electronics and Computer Science, University of Southampton, Southampton, SO17 1BJ, UK
}

\begin{abstract}
Electroporation is the creation of pores in a cell by applying an external electrical field and creating a voltage in the range of $200 \mathrm{mV}-1 \mathrm{~V}$ across the cell's membrane. This process is used intentionally in order to introduce chemicals, drugs or DNA into a cell. A model was created to determine whether electroporation would occur during the use of the signals typically used during functional electrical stimulation of skin and if it occurs what effect it has on the overall impedance of skin. Results from the simulation show that there is a threefold reduction in the electrical impedance of the cells.
\end{abstract}

\section{Introduction}

Being able to determine the electrical properties of the body has a number of practical uses. Knowing how a signal changes when it is sent through the skin or how it is altered when recorded from the body are relevant in disciplines such as biochemistry and biophysics as being highly relevant to many techniques used in medicine. A number of techniques for diagnosis and rehabilitation can be carried out safely and non-invasively by using information obtained from the electrical properties of skin[1-3].

One technique that involves sending electrical signals non-invasively into the body is called functional electrical stimulation (FES). In order to cause a muscle to move voluntarily a signal is sent from the brain to motor neurons through the nervous system[1,4]. When a signal is received at a motor neuron, a contraction occurs in the muscle fibres connected to the neuron, with the muscle fibres and motor neuron being collectively called a motor unit. FES involves attaching electrodes to the body and passing a current through them in order to cause contractions in otherwise paralysed muscles. Typically this is done through targeting the signal at nerves cells or at the junction between nerves and muscles in order to cause muscles to contract instead of stimulating the muscles directly. The current stimulates the nerve cell sending a signal to the central nervous system which causes the associated muscle fibres to contract. This is known as the $\mathrm{H}$ reflex. This process will produce signals from the activation of the muscle fibres known as M-waves. A typical type of signal used for FES is shown in figure 1. 
An example scenario where FES is used is for people who have suffered a stroke resulting in foot drop. Foot drop is a muscular weakness or paralysis that prevents the toes from lifting correctly and causing them to drag during the swing phase of the gait cycle[5].

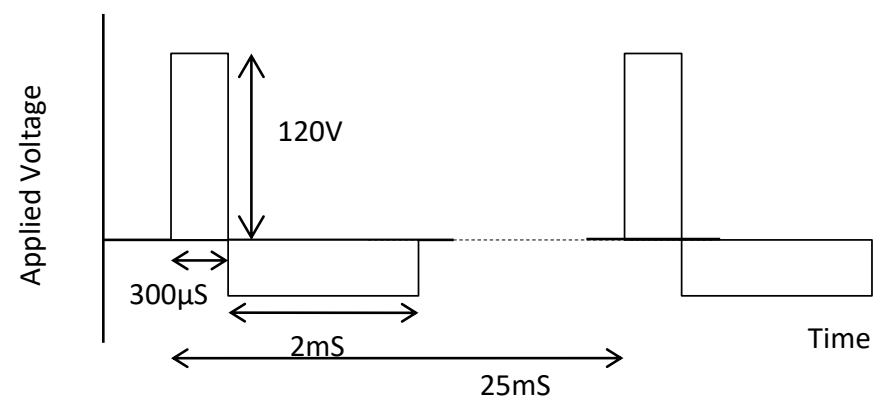

Figure 1 An illustration of a waveform used in FES. A typical waveform will have a frequency of $20-40 \mathrm{~Hz}$, amplitude of $100 \mathrm{~mA}$, applied voltage up to $120 \mathrm{~V}$ and pulse duration of up to $300 \mu \mathrm{S}$. A biphasic waveform is used to both introduce and remove charge i.e. charge balance to reduce irritation

\section{Skin Structure}

Skin is composed of two layers, the epidermis and the dermis. The epidermis is the outer most layer of skin and acts as a barrier. The epidermis is of particular importance because it has the largest impact on the overall impedance of skin. The epidermis is composed of a number of sub layers and is $95 \%$ composed of keratinocytes. Of these sub layers the stratum corneum (SC) has the largest influence on the overall impedance of skin. It is the outer most layer of the epidermis and is composed of terminally differentiated keratinocytes called corneocytes[6-8].

The corneocytes are very thin hexagonal or pentagonal cells. They contain very dense keratin and they overlap like a brick wall and are held together with the corneodesmosomes acting as rivets to maintain the rigid structure. Between the cells there is a complex array of lipids, ceramides, free fatty acids, cholesterol and cholesterol sulphate. Because they are dead cells and are poorly hydrated, they naturally have a very high electrical impedance compared to the cells in the lower levels of skin[8,9].

\section{Electroporation}

Electroporation is the process of changing the permeability of a cell membrane by applying an electrical field with a high enough intensity for a long enough period of time which creates a core in the membrane as shown in figure 2 . This process can either be reversible or irreversible, depending on the intensity and duration of the applied electric field. The signal used is also normally in the form of short pulses, which allows for a higher level of control over the electroporation process than a continuous signal. While it is thought that 
electroporation affects the electrical behaviour of skin it is not currently known how this phenomena affects FES[10-12].

FES requires a high level of accuracy to target specific nerves; it is important to have as much information as possible as to how a cell will react to an applied electric field. In order to do this without direct experimentation, models have been designed to determine a cell's electrical behaviour. A number of experiments have been performed in the literature using both individual cells as well as groups of cells in order to determine the exact nature of electroporation. However, this is still a growing area of research with many areas that have yet to be explored[13-17].

In order to cause a single cell to undergo electroporation, pulses on a microsecond to millisecond time scale must create an induced transmembrane voltage of around $200 \mathrm{mV}$ 1V. To create an electropore, an electrical field in the order of $10^{8} \mathrm{Vm}^{-1}$ must be applied[10,18].

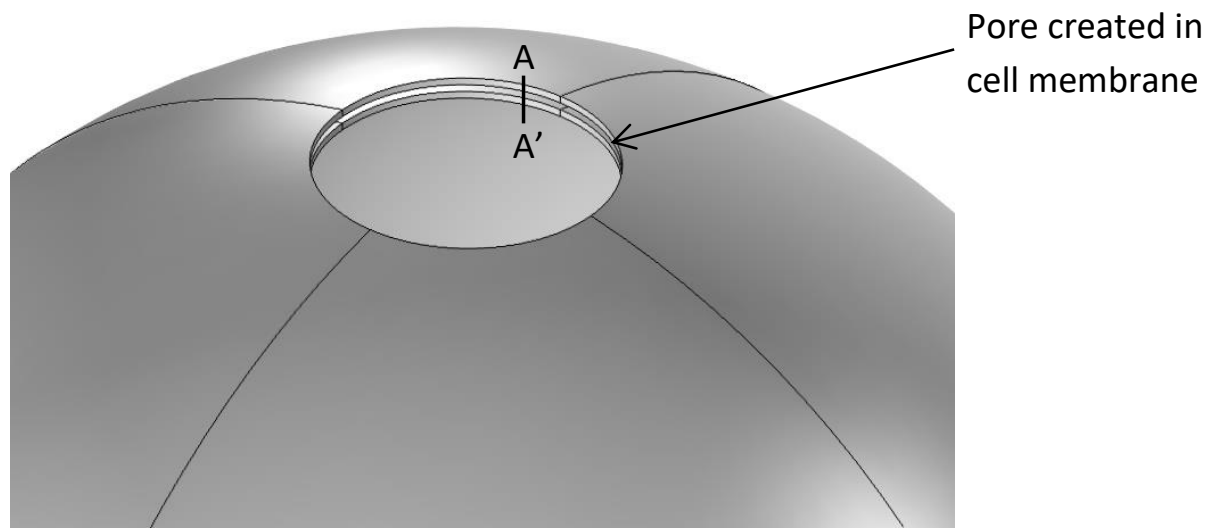

A
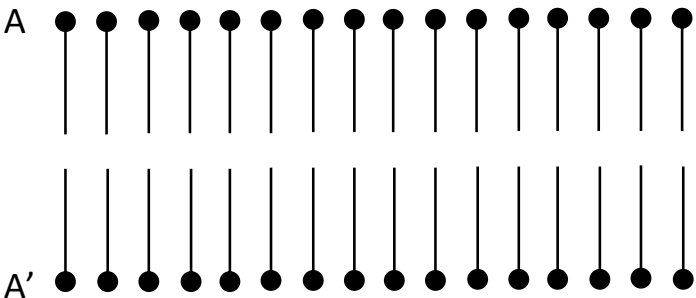

Bilipid layer before pore is created
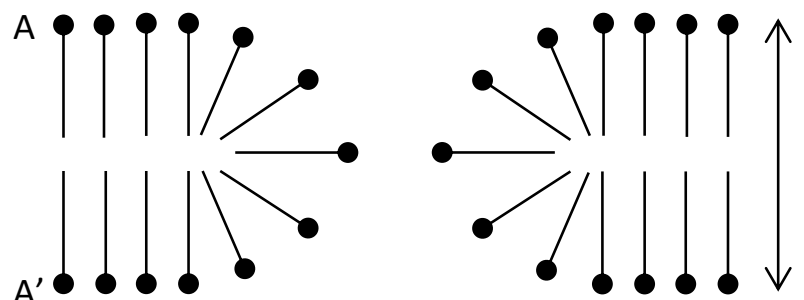

3-5 $\mathrm{nm}$

Figure 2 3D and 2D diagrams showing the creation of a pore due to an applied electric field

Research into electroporation is most often focused on the intentional creation of electropores, in particular for the targeted removal of cancer cells. Similarly studies looking at FES as well as the long term effect of electrodes do not mention electroporation as a possible factor for changes in skin 
impedance. The purpose of this study was to determine whether electroporation will occur during FES, where signals in the order of $100 \mathrm{~V}$ are commonly used.

\section{Materials and Methods}

A finite element model was developed to analyse the effect of electroporation on the impedance of skin. The model is composed of 10 layers of cells with physical dimensions and electrical properties corresponding to cells found in the SC, as well as 4 layers of larger cells with parameters selected to represent the remaining layers of epidermal cells. Surrounding theses layers of cells is an extracellular medium with properties matching those found in skin.

To create and simulate the model $\mathrm{COMSOL}^{\circledR} 5.2$ multiphysics modelling software was used. In order to simulate the pulses used in FES a time dependant study was created using the electric currents module. $\mathrm{COMSOL}^{\circledR}$ provides an approximate solution for a model given a set of starting parameters and a final desired error tolerance using finite element methods. The created model is divided into small segments known as elements and collectively called a mesh. $\mathrm{COMSOL}^{\circledR}$ uses a set of equations determined by the electric currents module to simulate the effects of the applied signal. Modules for mechanical and thermal effects were not included in the study. The reason for this that due to the size and time scale of the input signals being used is small enough that any thermal and mechanical effects would be negligible[13,20,21].

The overall geometry of the model can be seen in figure 3, showing the SC cells and the corneodesmosomes and lower layer epidermal cells but not the surrounding medium for visual clarity. Each element was then given a relative permittivity and electrical conductivity in keeping with the values for their biological counterparts, with the exact values shown in table 1. A mesh was not created for the cell membrane due to its thickness being only $4 \mathrm{~nm}$ and therefore requiring very small elements that greatly increase simulation run time. Instead the membrane was substituted with a boundary condition that represents that behaviour of the impedance of the membrane, which has been shown to be more accurate and require less computational power than by using large mesh elements[22]. 


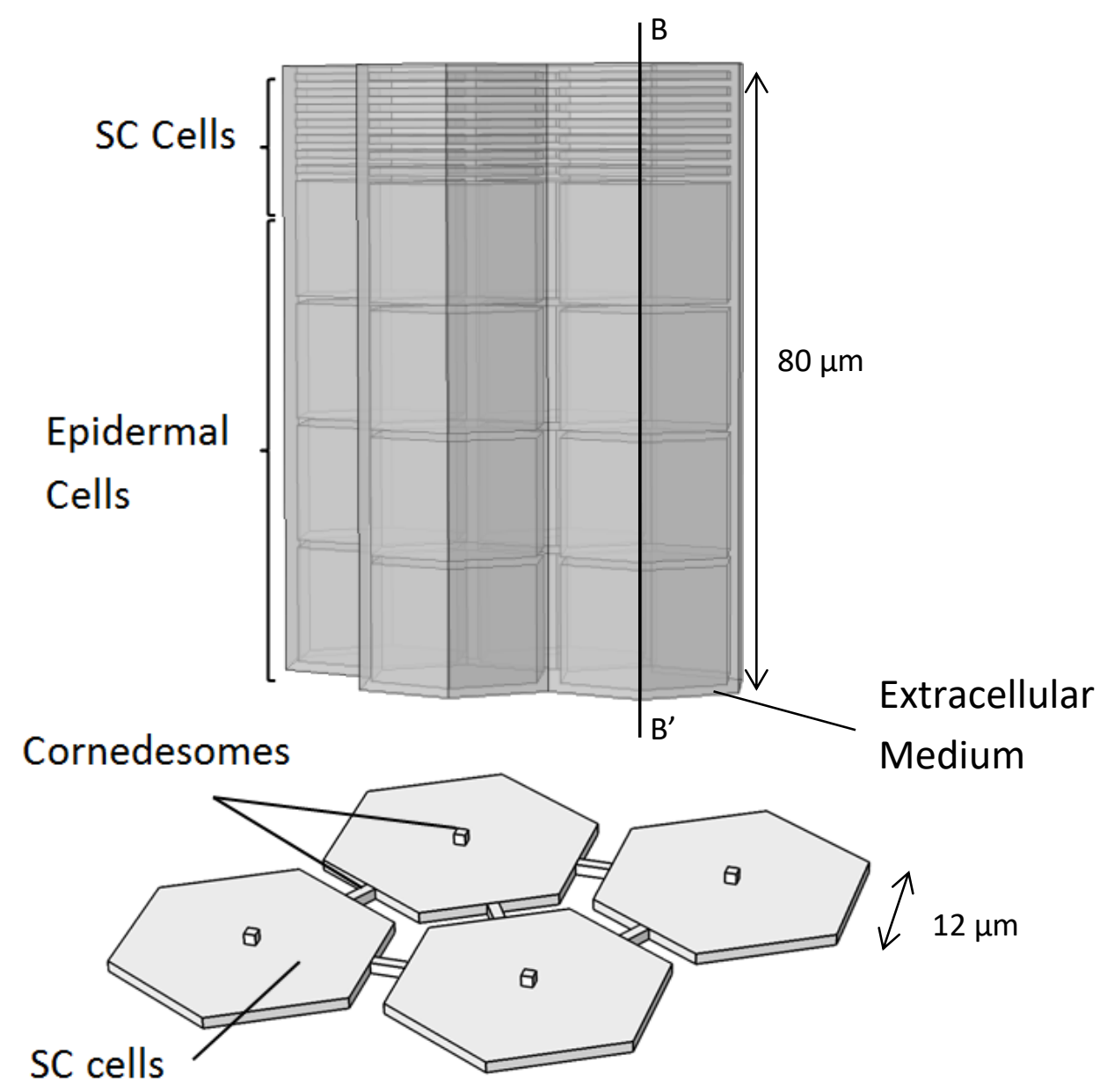

Figure 3 Geometry of the model showing the extracellular medium and the three types of cell used with one layer showing the cornedesomes

One side of the extracellular medium was set to be the input signal with the opposite side grounded. The remaining sides are set as electrical insulators.

The input was created using a rectangle function with peak voltages of $0.1 \mathrm{~V}, 10 \mathrm{~V}, 100 \mathrm{~V}$ and $1000 \mathrm{~V}$. The pulse had a period of $300 \mu$ s and a rising edge of $80 \mu \mathrm{s}$, an example signal is shown in figure 4 . These times were chosen so that the simulation would converge to a solution across all combinations of variables. The input voltages were simulated with membrane thicknesses of 3, 4 and $5 \mathrm{~nm}$, to cover the thickness shown in literature and with a threshold ITV required to create an electropore set to values between $0.2 \mathrm{~V}$ and $1 \mathrm{~V}$, with an interval of $0.1 \mathrm{~V}$. 


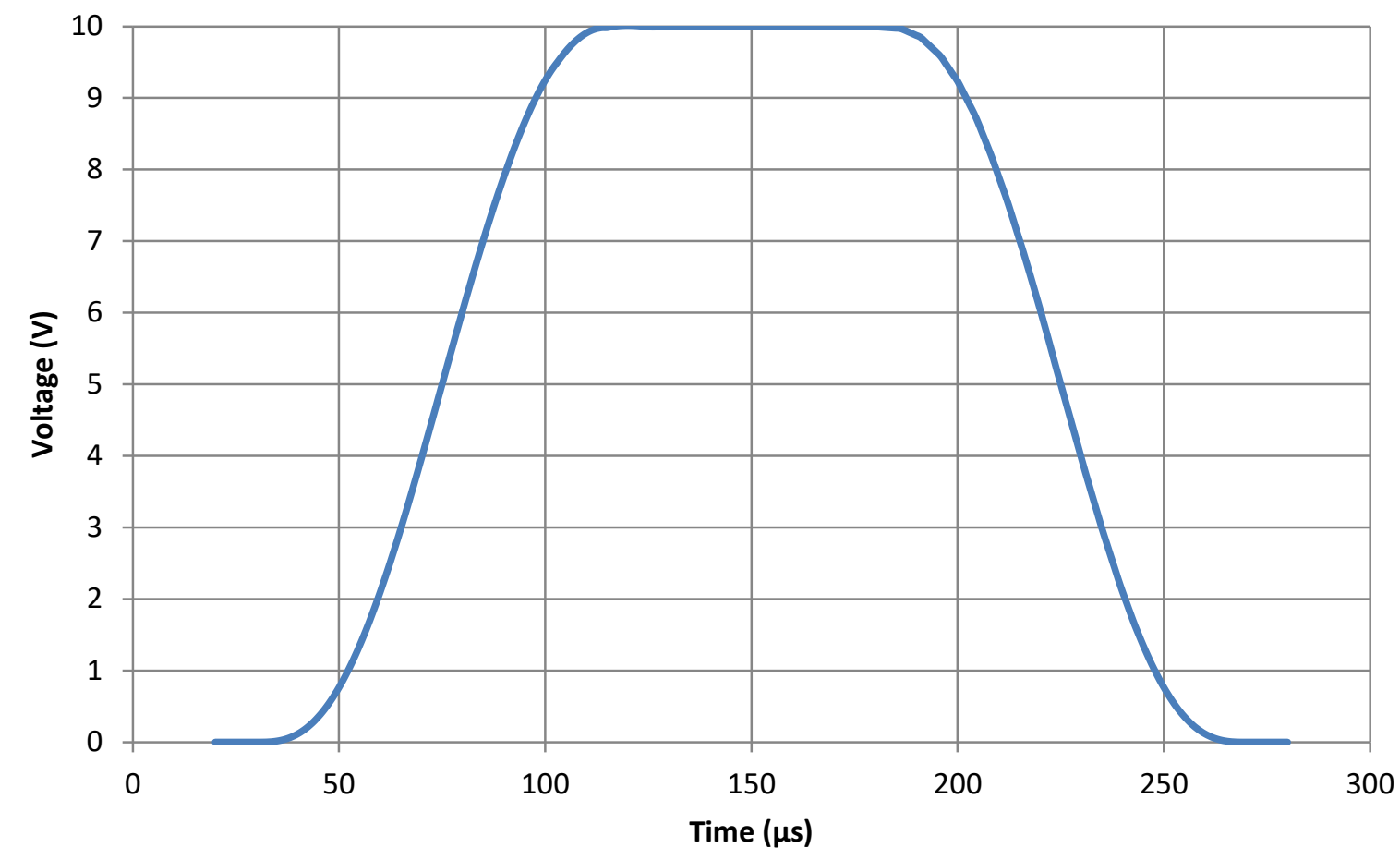

Figure 4, Input Voltage used in 10V simulation

To model the effect of electroporation a switching function was used to determine the conductivity of the cell membrane. When the voltage across a cell membrane is below a threshold ITV the conductivity is $1 \times 10^{-7} \mathrm{Sm}^{-1}$ and when the voltage is above the threshold it is $0.6 \mathrm{Sm}^{-1}$ (Table 1).

Table 1 Parameters for the single cell model

\begin{tabular}{cccc}
\hline Object & $\begin{array}{c}\text { Dimensions } \\
(\mu \mathrm{m})\end{array}$ & $\begin{array}{c}\text { Relative } \\
\text { permittivity }\end{array}$ & $\begin{array}{c}\text { Conductivity } \\
\left(\mathrm{Sm}^{-1}\right)\end{array}$ \\
\hline $\begin{array}{c}\text { Extracellular } \\
\text { medium }\end{array}$ & $34 \times 34 \times 80$ & 72 & 1.1 \\
$\begin{array}{c}\text { Cytoplasm } \\
\text { SC Cytoplasm }\end{array}$ & $30 \times 30 \times 30$ & 86 & 0.6 \\
Cell & $30 \times 30 \times 1$ & 86 & $1 \times 10^{-7}$ \\
Membrane & & 90 & $1 \times 10^{-7}$ \\
\hline
\end{tabular}




\section{Results}

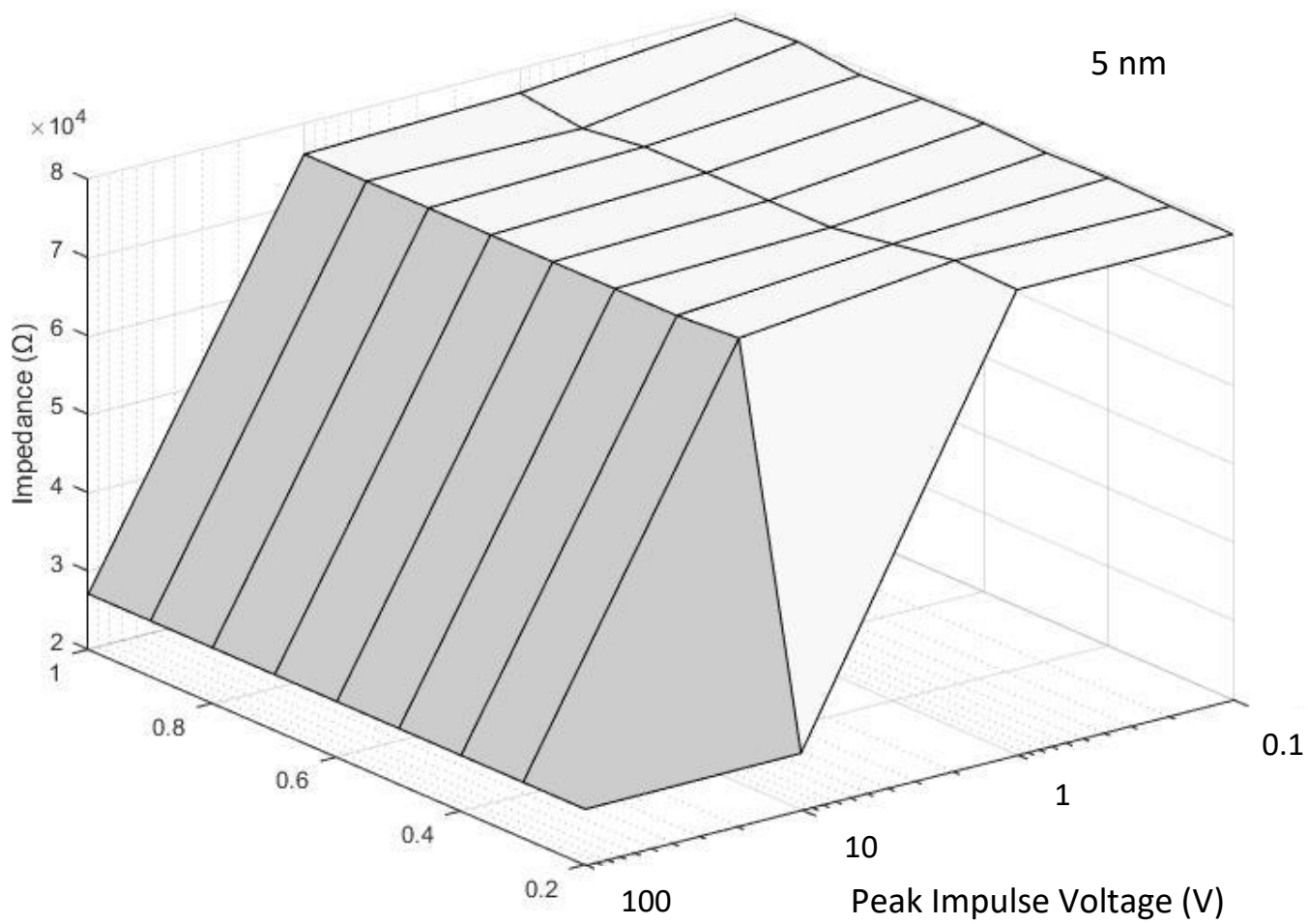

ITV Threshold (V)

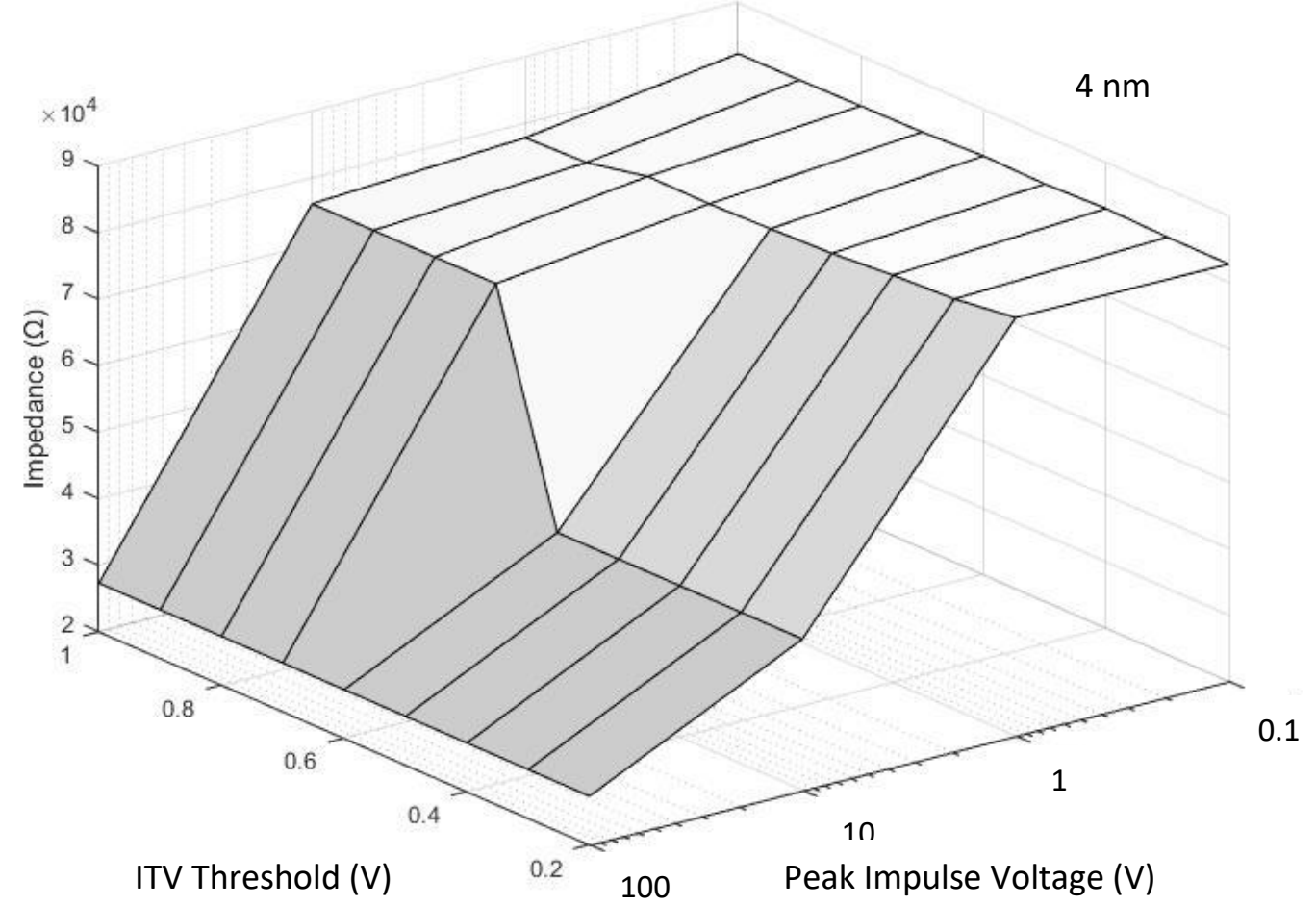




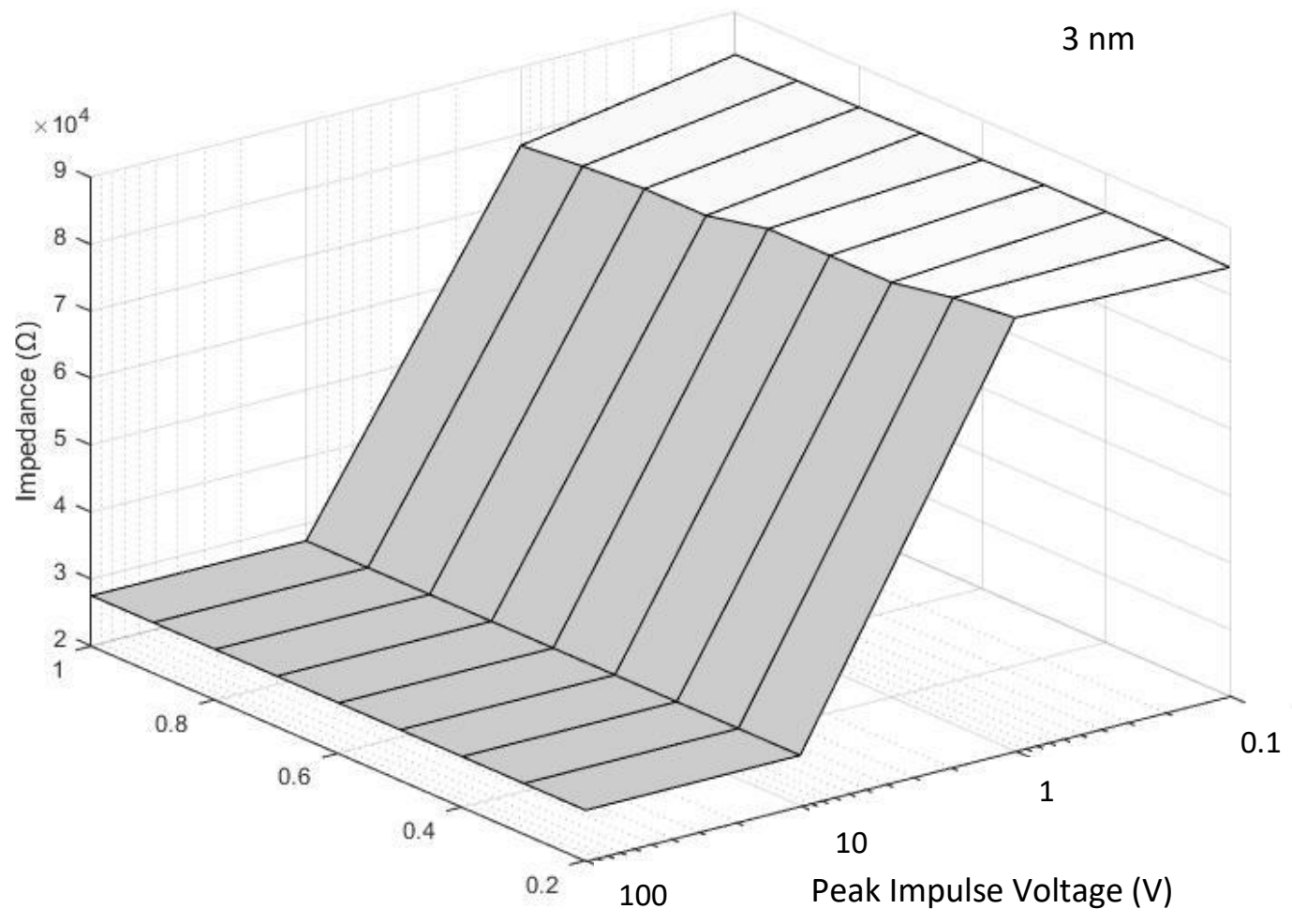

ITV Threshold (V)

Figure 5 Impedance measured at time step $150 \mu \mathrm{s}$, the centre of the pulse shown in figure 4 , showing changes due to peak impulse voltage (using a logarithmic scale) and ITV threshold voltage required to create and electropore. The surface at the top plot shows the impedance with a membrane thickness of $5 \mathrm{~nm}$, the middle plot with a membrane thickness of $4 \mathrm{~nm}$ and the bottom plot with a membrane thickness of $3 \mathrm{~nm}$ 


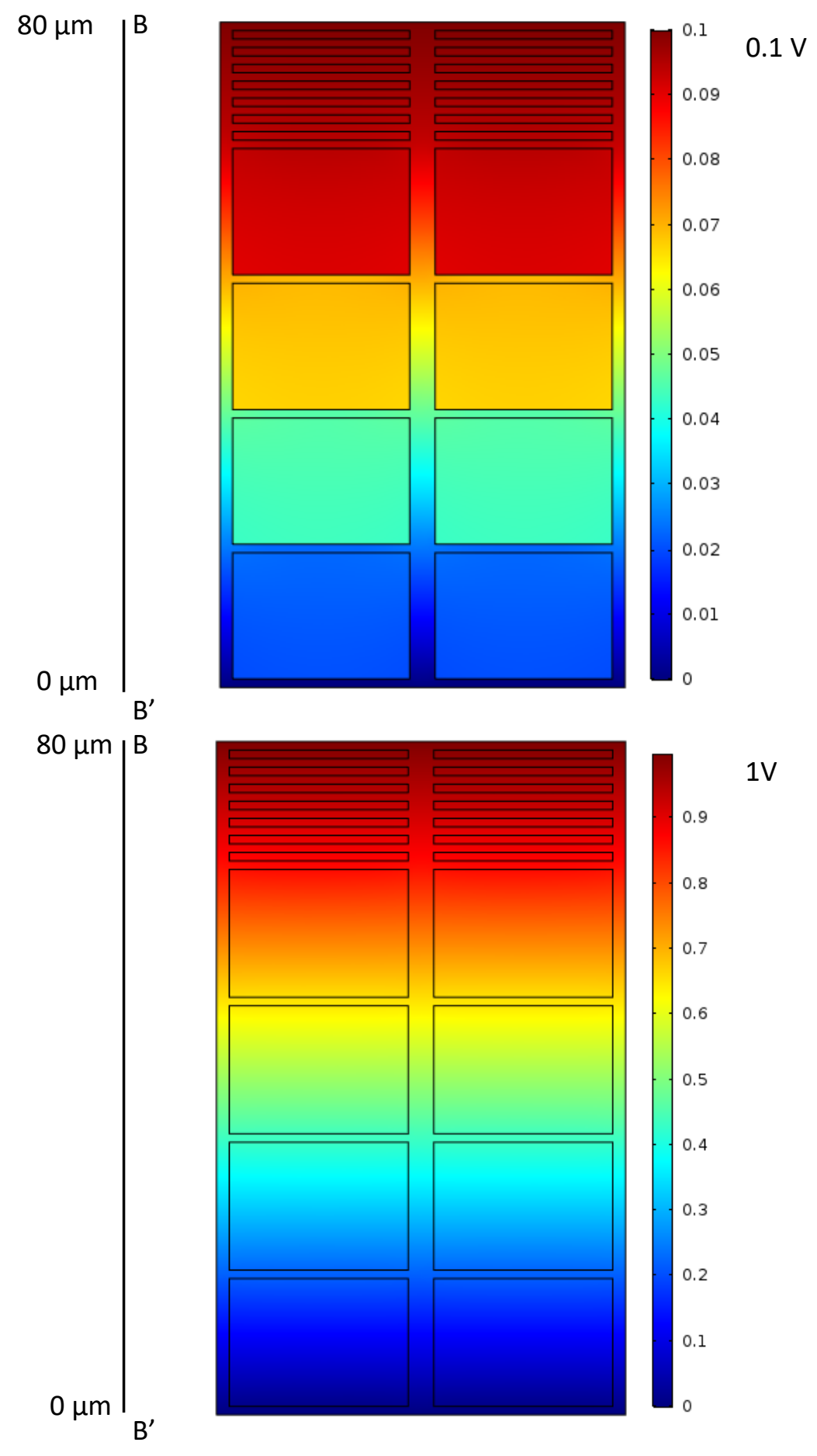




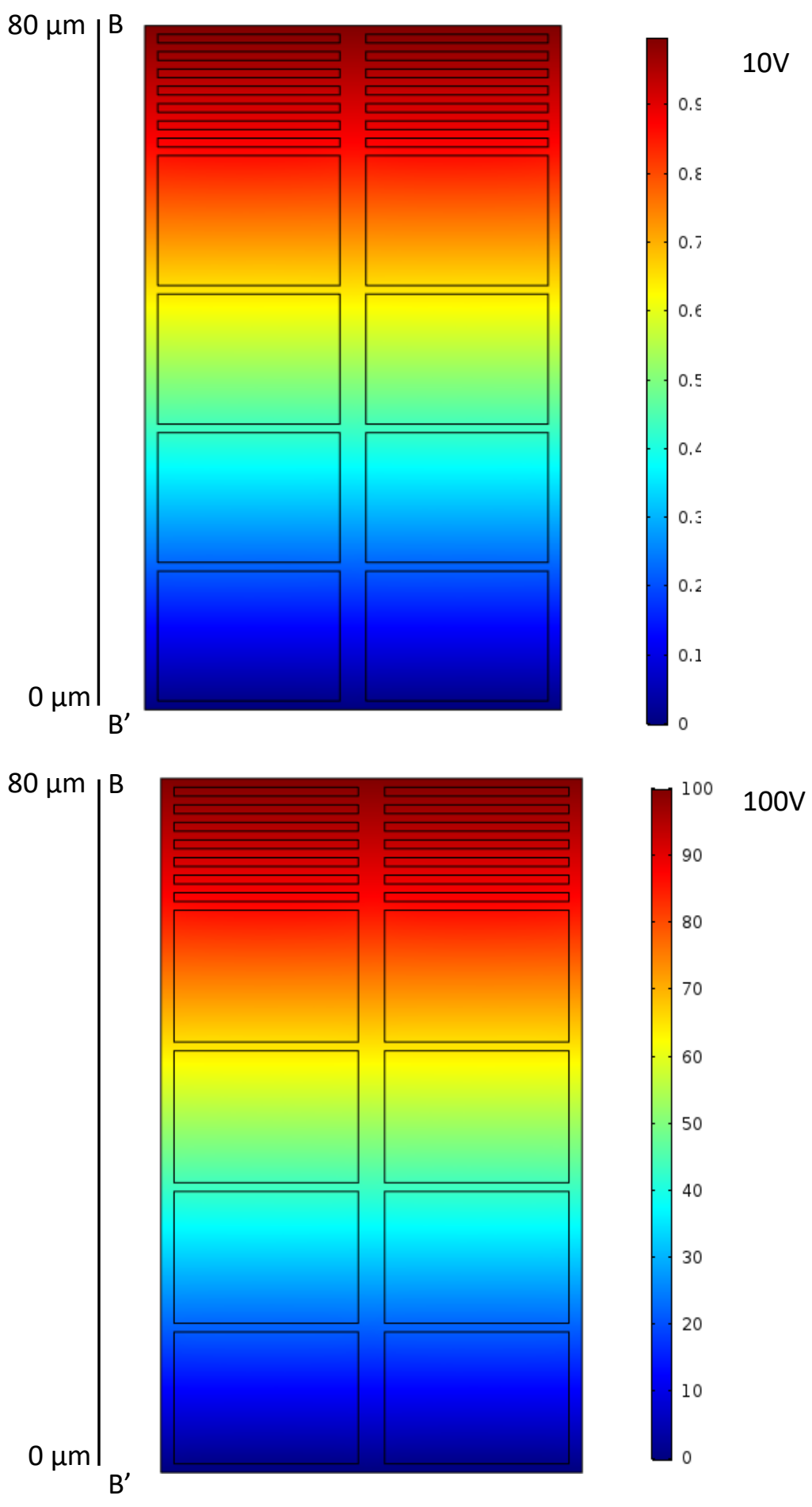

Figure 6 Voltage gradients across the model at $150 \mu$ s with different peak impulse voltages. The plots show a cut in a plane of the model at the section $B$ to $B^{\prime}$ as shown in figure 3 with a $0.1 \mathrm{~V}, 1 \mathrm{~V}, 10 \mathrm{~V}$ and $100 \mathrm{~V}$ peak voltages. Shown on the right hand side is the colour scale for voltage in each cell with respect to the zero voltage at the base of the cells ( $\left.B^{\prime}\right)$ 


\section{Discussion}

The results of the simulation, (Figure 5), show that for most of the parameter values electropores are being created. This suggests that in general there will be electropores being formed when FES is used, especially since typical FES signals have amplitude of at least 100V, a value at which electropores were formed for all values of the ITV value or the membrane thickness that were simulated. Figure 5 shows that when the peak impulse voltage is $0.1 \mathrm{~V}$ there are no electropores being formed and when the input amplitude is $10 \mathrm{~V}$ pores are formed at all membrane thicknesses and when the amplitude is $1 \mathrm{~V}$ pores are formed with a membrane thickness of either $3 \mathrm{~nm}$ or $4 \mathrm{~nm}$, depending on the ITV threshold.

The effect on impedance of electropores being created is substantial, with a drop from $76 \mathrm{k} \Omega$ to $22 \mathrm{k} \Omega$. This shows that if voltage signals of at least $1 \mathrm{~V}$ are used and the threshold voltage is $0.2 \mathrm{~V}$ there will be a huge impact on the impedance of skin. The top plot in figure 6 shows that when the peak input voltage is $0.1 \mathrm{~V}$ there is no change in voltage across the cells, shown by the colour not changing within the cells, which shows that there is only a small current flowing through the cells and creating the overall impedance of $76 \mathrm{k} \Omega$. When the peak voltage is $1 \mathrm{~V}, 10 \mathrm{~V}$ or $100 \mathrm{~V}$ pores are formed in the membrane, 'which can be seen from the colour gradient changing in the same way as the surrounding cell medium, meaning that current can now flow freely through the membrane, greatly reducing the skin's overall impedance.

\section{Conclusion}

The simulation shows that using the experimentally determined properties of skin cells and the equation used to model a localised drop in membrane impedance; electropores will form in the voltage ranges that would be expected from experimental observations, from $1 \mathrm{~V}$ and upwards, depending on the thickness of the membrane and the ITV threshold.

The results of the model show that when electropores are formed there is a significant decrease in overall skin impedance, with impedances of $76 \mathrm{k} \Omega$ at $0.1 \mathrm{~V}, 74 \mathrm{k} \Omega, 24 \mathrm{k} \Omega$ at $10 \mathrm{~V}$ and $22 \mathrm{k} \Omega$ at $100 \mathrm{~V}$, membrane thickness of $3 \mathrm{~nm}$ and ITV of $0.2 \mathrm{~V}$. The creation of pores occurs when input voltages above $1 \mathrm{~V}$ were used depending on the membrane thickness and exact input voltage used, meaning that during the typical use of FES the effects of electroporation should be taken into account. This can be done by assuming a significant decrease in impedance almost instantaneously after a signal is applied or by using a signal with a peak voltage lower than $1 \mathrm{~V}$. 
[1] Lynch C L and Popovic M R 2008 Functional Electrical Stimulation IEEE Control Syst. 28 40-50

[2] Weitzen R, Epstein N, Shoenfeld $Y$ and Zimlichman E 2007 Diagnosing Diseases by Measurement of Electrical Skin Impedance Ann. N. Y. Acad. Sci. 1109 185-92

[3] Martinsen $\varnothing$ G, Grimnes S and Karlsen J 1995 Electrical Methods for Skin Moisture Assessment Skin Pharmacol. Physiol. 8 237-45

[4] Popovic D and Sinkjaer T 2012 Control of Movement for the Physically Disabled: Control for Rehabilitation Technology (Springer Science \& Business Media)

[5] Rushton D N 1997 Functional electrical stimulation Physiol. Meas. 18241

[6] Baroni A, Buommino E, De Gregorio V, Ruocco E, Ruocco V and Wolf R 2012 Structure and function of the epidermis related to barrier properties Clin. Dermatol. 30 257-62

[7] Elias P M 2012 Structure and Function of the Stratum Corneum Extracellular Matrix J. Invest. Dermatol. 132 2131-3

[8] Lai-Cheong J E and McGrath J A 2009 Structure and function of skin, hair and nails Medicine (Baltimore) 37 223-6

[9] Menon G K, Cleary G W and Lane M E 2012 The structure and function of the stratum corneum Int. J. Pharm. 435 3-9

[10] Apollonio F, Liberti M, Marracino P and Mir L 2012 Electroporation mechanism: Review of molecular models based on computer simulation 2012 6th European Conference on Antennas and Propagation (EUCAP) 2012 6th European Conference on Antennas and Propagation (EUCAP) pp 356-8

[11] Arias L R, Perry C A and Yang L 2010 Real-time electrical impedance detection of cellular activities of oral cancer cells Biosens. Bioelectron. 25 2225-2231

[12] Bai S-J and Prinz F B 2011 In vivo electrochemical impedance measurement on single cell membrane Microelectron. Eng. 88 3094-100

[13] Hjouj M and Rubinsky B 2013 Electroporation Image-Guided Cancer Therapy ed D E Dupuy, Y Fong and W N McMullen (Springer New York) pp 21-36

[14] Boucsein W 2012 Electrodermal Activity (Springer)

[15] Benedek M and Kaernbach C 2010 A continuous measure of phasic electrodermal activity J. Neurosci. Methods 190 80-91

[16] Neumann E, Schaefer-Ridder M, Wang Y and Hofschneider P H 1982 Gene transfer into mouse lyoma cells by electroporation in high electric fields. EMBO J. 1 841-5 
[17] Gehl J and Mir L M 1999 Determination of Optimal Parameters for in Vivo Gene Transfer by Electroporation, Using a Rapid in Vivo Test for Cell Permeabilization Biochem. Biophys. Res. Commun. 261 377-80

[18] Chen C, Smye S W, Robinson M P and Evans J A 2006 Membrane electroporation theories: a review Med. Biol. Eng. Comput. 44 5-14

[19] Jiang C, Davalos R V and Bischof J C 2015 A Review of Basic to Clinical Studies of Irreversible Electroporation Therapy IEEE Trans. Biomed. Eng. 62 4-20

[20] Cifra M, Fields J Z and Farhadi A 2011 Electromagnetic cellular interactions Prog. Biophys. Mol. Biol. 105 223-246

[21] Criswell E 2010 Cram's introduction to surface electromyography (Jones \& Bartlett Publishers)

[22] Pucihar G, Kotnik T, Valič B and Miklavčič D 2006 Numerical determination of transmembrane voltage induced on irregularly shaped cells Ann. Biomed. Eng. 34 642652 